УДК $517.26,519.7$

\author{
P. S. SENIO
}

\title{
MATRIX REPRESENTATION OF TAYLOR'S FORMULA FOR MAPPINGS IN FINITE DIMENSIONAL SPACES
}

\begin{abstract}
P. S. Senio. Matrix representation of Taylor's formula for mappings in finite dimensional spaces, Mat. Stud. 51 (2019), 92-106.

In this paper, the matrix representations of derivatives, differentials and Taylor's formulas for functionals and mappings are presented. The behavior law of intermediate points in the formula's remainder terms is found for shrinking the expansion interval of mapping to a point. This, in particular, allows one to analyze the local uncertainties in the remainder terms of Taylor's formula and to develop and explore the new effective methods for solving systems of nonlinear algebraic equations.
\end{abstract}

1. Introduction. If some method of solving a given problem is iterative, then in the case of its convergence to the solution, the distance from the approximate values to the solution tends to zero. Since many of these methods use Taylor's formula, it is important to clarify the behavior of the intermediate point in the remainder of this formula while shrinking the expansion interval of mapping to the point. However, with the increase of the space dimension and the order of derivatives and differentials, the complexity of Taylor's formula increases rapidly. This complicates its application and analysis of the uncertainty in its remainders.

For example, for a mapping $F: \mathbb{R}^{n} \rightarrow \mathbb{R}^{m}(m>1)$ the mean value theorem according to known statement from the one dimensional case is a false statement. Although there are three approaches $[1,2]$ to solve this problem, the remainder term of Taylor's formula has not been estimated [1].

There are three manuals considering general Taylor's formula for mapping from a normed space to another normed space [6, p. 599], [8, p. 87], [7, p. 715]. They also propose some estimates of the remainder term.

To do this, a specific form of $k$-linear mappings of the $k$-th order derivatives and corresponding differentials of mapping $F$ must be found. This gives us the matrix representation of Taylor's formula. Hence, the corresponding operations with such $k$-linear mappings can be used to analyze the behavior of the intermediate point in the remainder of this formula while shrinking the expansion interval of mapping to the point.

2. A matrix representation of derivative maps in finite-dimensional spaces. We need the following theorem.

2010 Mathematics Subject Classification:41A58, 46N10, 46T20.

Keywords: functional and mapping derivatives; functional and mapping differentials; Taylor's formula for functionals and mappings; remainder terms of Taylor's formula.

doi:10.15330/ms.51.1.92-106

(C) P. S. Senio, 2019 
Theorem 1 ([1]). Let a mapping $F: \mathbb{R}^{n} \rightarrow \mathbb{R}^{m}$ be $k$ times differentiable. Then mapping between $K(\underbrace{\mathbb{R}^{n} \times \mathbb{R}^{n} \times \cdots \times \mathbb{R}^{n}}_{k \text { times }}, \mathbb{R}^{m})$ and $\underbrace{L\left(\mathbb{R}^{n}, L\left(\mathbb{R}^{n}, \cdots, L\left(\mathbb{R}^{n}, \mathbb{R}^{m}\right)\right)\right)}_{k \text { times }}$, which maps each element $A \in L\left(\mathbb{R}^{n}, L\left(\mathbb{R}^{n}, \cdots, L\left(\mathbb{R}^{n}, \mathbb{R}^{m}\right)\right)\right)$ to $K \in K\left(\mathbb{R}^{n} \times \mathbb{R}^{n} \times \cdots \times \mathbb{R}^{n}, \mathbb{R}^{m}\right)$ by the formula

$$
K\left(x^{(1)}, x^{(2)}, \cdots, x^{(k)}\right)=\left(\left(\left(\left(A x^{(1)}\right) x^{(2)}\right) \cdots\right) x^{(k-1)}\right) x^{(k)}
$$

is linear, isometric and one-to-one. Besides, the image of the space $L\left(\mathbb{R}^{n}, L\left(\mathbb{R}^{n}, \cdots\right.\right.$, $\left.\left.L\left(\mathbb{R}^{n}, \mathbb{R}^{m}\right)\right)\right)$ is the whole space $K\left(\mathbb{R}^{n} \times \mathbb{R}^{n} \times \cdots \times \mathbb{R}^{n}, \mathbb{R}^{m}\right)$.

Corollary 1 ([1]). $k$-th order derivative of mapping $F$ can be considered as an element of space $K(\underbrace{\mathbb{R}^{n} \times \mathbb{R}^{n} \times \cdots \times \mathbb{R}^{n}}_{k \text { times }}, \mathbb{R}^{m})$.

Lemma 1. Let mapping $F(x)$ with components $f_{1}, f_{2}, \ldots, f_{m}$ be Gateaux differentiable and its derivative is linear mapping $A\left(A=\left(a_{i j}\right)\right)$. Then its derivative is a column vector $\left(f_{1}^{\prime}, f_{2}^{\prime}, \cdots, f_{m}^{\prime}\right)^{T}$ of component derivatives (Jacobi matrix).

Proof. Let $\Delta x=e_{j}$, where $e_{j}$ is a $j$-th coordinate vector. Then by the definition of the Gateaux derivative $a_{i j}(i, j=\overline{1, m})$ of the mapping $F(x)$ one has

$$
\lim _{t \rightarrow 0} \frac{\left\|f_{i}\left(x+t \cdot e_{j}\right)-f_{i}(x)-t a_{i j}\right\|}{t}=0,
$$

that is, the derivative of the mapping $F(x)$ is linearly ordered by the first index of the set of derivatives of its components. Lemma 1 is proved.

Remark 1. Since the linear mappings $\mathbb{R}^{n}$ onto $\mathbb{R}^{m}$ form a linear normalized space, the components of such mappings form column vectors. Therefore, before the differentiation of the mappings in the case when their components form row vectors, each such a vector must be pre-transposed into a corresponding column vector.

We use a formal notation $\left(A_{1} A_{2} \ldots A_{p}\right)^{T}$ instead of $\left(\begin{array}{c}A_{1} \\ A_{2} \\ \vdots \\ A_{n}\end{array}\right)$, where $A_{j}$ is some matrix or block of matrices.

Let $F: \mathbb{R}^{n} \rightarrow \mathbb{R}^{m}$ be a mapping with components $F=\left(f_{1}, f_{2}, \cdots, f_{m}\right)$. Assume that it is the Gateaux $k$-times differentiable mapping. We denote

$$
f_{l, x_{(1)} x_{(2)} \ldots x_{(k)}}=\frac{\partial^{k}}{\partial x_{(1)} \partial x_{(2)} \ldots \partial x_{(k)}} f_{l}\left(x_{1}, x_{2}, \ldots, x_{n}\right),
$$

where $x_{(j)} \in\left\{x_{1}, x_{2}, \ldots, x_{n}\right\},(i, j, p=\overline{1, n}, l=\overline{1, m}, k=1,2, \ldots)$, and

$$
S_{f, r}^{(k)}(x)=\left(\begin{array}{cccc}
g_{i j 1, x_{1}} & g_{i j 1, x_{2}} & \cdots & g_{i j 1, x_{n}} \\
g_{i j 2, x_{1}} & g_{i j 2, x_{2}} & \cdots & g_{i j 2, x_{n}} \\
\cdots & \cdots & \ddots & \cdots \\
g_{i j n, x_{1}} & g_{i j n, x_{2}} & \cdots & g_{i j n, x_{n}}
\end{array}\right) \quad(k=1,2, \cdots)
$$


where $g_{i j q}(x)$ is the $q$-th element of the $j$-th row of the generating matrix $S_{f, i}^{(k-1)}(x)$ and $g_{i j q, x_{p}}(x)=\frac{\partial}{\partial x_{p}} g_{i j q}(x)$; the generating matrix $S_{f, 1}^{(2)}$ (i.e. for $k=2$ ) is the Hessian matrix of the mapping $f: \mathbb{R}^{n} \rightarrow \mathbb{R}$. A matrix $S_{f, j}^{(k)}(x),\left(i=\overline{1, n^{k-2}} ; j, p, q=\overline{1, n} ; k=2,3, \ldots\right)$ is called $i$-th generating matrix of the $k$-th order derivative of functional $f$, created by $i$-th row of $j$-th generating matrix $S_{j, r}^{(k-1)}(x),(r=(i-1) n+j)$ of $(k-1)$-th order derivative of this functional.

The obtained results give an opportunity to formulate and prove the following theorem.

Theorem 2. Let a functional $f: \mathbb{R}^{n} \rightarrow \mathbb{R}^{1}$ be $k$-times differentiable. Then the $k$-linear structured hypermatrix

$$
F_{f}^{(k)}(x)=\left(\begin{array}{c}
\left(\begin{array}{c}
S_{f, 1}^{(k)}(x) \\
\vdots \\
S_{f, n}^{(k)}(x)
\end{array}\right) \\
\vdots \\
\left(\begin{array}{c}
S_{f, n+1}^{(k)}(x) \\
\vdots \\
S_{f, n^{2}}^{(k)}(x)
\end{array}\right) \\
\vdots \\
\left(\begin{array}{c}
S_{f, n\left(n^{k-4}-1\right)+1}^{(k)}(x) \\
\vdots \\
S_{f, n^{k-3}}^{(k)}(x) \\
\vdots \\
\left(\begin{array}{c}
S_{f, n\left(n^{k-3}-1\right)+1}^{(k)}(x) \\
\vdots \\
S_{f, n^{k-2}}^{(k)}(x)
\end{array}\right)
\end{array}\right)
\end{array}\right)
$$

is $k$-th derivative $f^{(k)}(x)$ of functional $f$ at a point $x \in \mathbb{R}^{n}$.

Proof. The proof is based by the method of mathematical induction.

Let $k=1$. Then the derivative of the $y=f(x)=f\left(x_{1}, x_{2}, \ldots, x_{n}\right)$ is the transposed gradient of this function at $x$, that is, $f^{\prime}(x)=\left(f_{x_{1}}(x), f_{x_{2}}(x), \cdots, f_{x_{n}}(x)\right)$.

Let $k=2$. By Corollary 1 the derivative $f^{\prime}(x)=\left(f_{x_{1}}(x), f_{x_{2}}(x), \ldots, f_{x_{n}}(x)\right)$ is the element of the space $K_{1}\left(\mathbb{R}^{n}, \mathbb{R}^{n}\right)$. But the mapping $F_{f}^{\prime}(x)$ of this space has components $f_{x_{1}}(x), f_{x_{2}}(x), \cdots, f_{x_{n}}(x)$. Hence, $F_{f}^{\prime}(x)=\left(f_{x_{1}}(x), f_{x_{2}}(x), \cdots, f_{x_{n}}(x)\right)^{T}$ and $F_{f}^{\prime \prime}(x)=$ $\left(F_{f}^{\prime}(x)\right)^{\prime}$. Then by Lemma $1 F_{f}^{\prime \prime}(x)=\left(f_{x_{1}}^{\prime}(x), f_{x_{2}}^{\prime}(x), \cdots, f_{x_{n}}^{\prime}(x)\right)^{T}$.

The components $f_{x_{1}}(x), f_{x_{2}}(x), \cdots, f_{x_{n}}(x)$ are functionals. Therefore,

$$
F_{f}^{\prime \prime}(x)=\left(\begin{array}{c}
\left(f_{x_{1}}(x)\right)^{\prime} \\
\left(f_{x_{2}}(x)\right)^{\prime} \\
\vdots \\
\left(f_{x_{n}}(x)\right)^{\prime}
\end{array}\right)=\left(\begin{array}{cccc}
\frac{d}{d x_{1}} f_{x_{1}}(x) & \frac{d}{d x_{2}} f_{x_{1}}(x) & \cdots & \frac{d}{d x_{n}} f_{x_{1}}(x) \\
\frac{d}{d x_{1}} f_{x_{2}}(x) & \frac{d}{d x_{2}} f_{x_{2}}(x) & \cdots & \frac{d}{d x_{n}} f_{x_{2}}(x) \\
\vdots & \vdots & \ddots & \vdots \\
\frac{d}{d x_{1}} f_{x_{n}}(x) & \frac{d}{d x_{2}} f_{x_{n}}(x) & \cdots & \frac{d}{d x_{n}} f_{x_{n}}(x)
\end{array}\right)=
$$




$$
=\left(\begin{array}{cccc}
f_{x_{1} x_{1}}(x) & f_{x_{1} x_{2}}(x) & \cdots & f_{x_{1} x_{n}}(x) \\
f_{x_{2} x_{1}}(x) & f_{x_{2} x_{2}}(x) & \cdots & f_{x_{2} x_{n}}(x) \\
\vdots & \vdots & \ddots & \vdots \\
f_{x_{n} x_{1}}(x) & f_{x_{n} x_{2}}(x) & \cdots & f_{x_{n} x_{n}}(x)
\end{array}\right)=H_{f}(x) .
$$

Since $H_{f}(x)=S_{f, 1}^{(3)}(x)$, taking into account (5), we obtain the equality $F_{f}^{(3)}(x)=$ $\left(S_{f, 1}^{(2)}(x)\right)$. The mapping $F_{f}^{\prime \prime}(x)$ is an element of the space $K_{2}\left(\mathbb{R}^{n} \times \mathbb{R}^{n}, \mathbb{R}^{n}\right)$. According to Corollary 1, this element can be considered as the second derivative $f^{\prime \prime}(x)$ of the functional $y=f(x)$. Here, it is taken into account that by Theorem 1 the image of the space $L\left(\mathbb{R}^{n}, L\left(\mathbb{R}^{n}, \cdots, L\left(\mathbb{R}^{n}, \mathbb{R}^{m}\right)\right)\right)$ is the entire space $K\left(\mathbb{R}^{n} \times \mathbb{R}^{n} \times \cdots \times \mathbb{R}^{n}, \mathbb{R}^{m}\right)$.

The proof of formula (4) starts with the next step.

Let $k=3$. The mapping $F_{f}^{\prime \prime}(x)$ of the space $K_{2}\left(\mathbb{R}^{n} \times \mathbb{R}^{n}, \mathbb{R}^{n}\right)$ has $n$ components

$$
\begin{gathered}
\left(\begin{array}{cccccc}
f_{x_{1} x_{1}}(x) & f_{x_{1} x_{2}}(x) & \cdots & f_{x_{1} x_{n}}(x)
\end{array}\right),\left(\begin{array}{llll}
f_{x_{2} x_{1}}(x) & f_{x_{2} x_{2}}(x) & \cdots & f_{x_{2} x_{n}}(x)
\end{array}\right) \\
\left(\begin{array}{llll}
f_{x_{n} x_{1}}(x) & f_{x_{n} x_{2}}(x) & \cdots & f_{x_{n} x_{n}}(x)
\end{array}\right)
\end{gathered}
$$

where each components is the corresponding derivative $f_{x_{1}}(x), f_{x_{2}}(x), \cdots, f_{x_{n}}(x)$ of the first derivative of $F_{f}^{\prime}(x)$. Since $F_{f}^{\prime \prime \prime}(x)=\left(F_{f}^{\prime \prime}(x)\right)^{\prime}$, by Lemma 1 we have

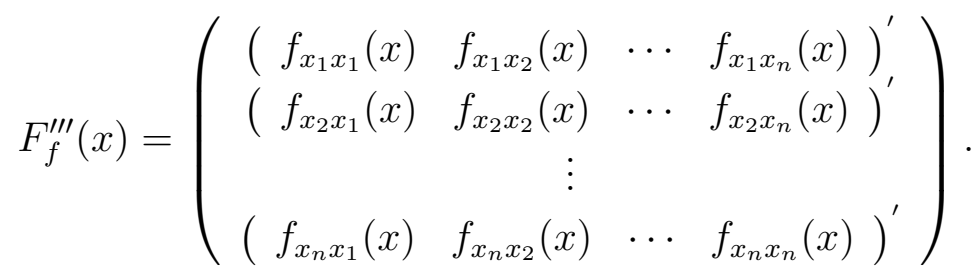

Consequently applying Corollary 1, differentiating each row, we obtain

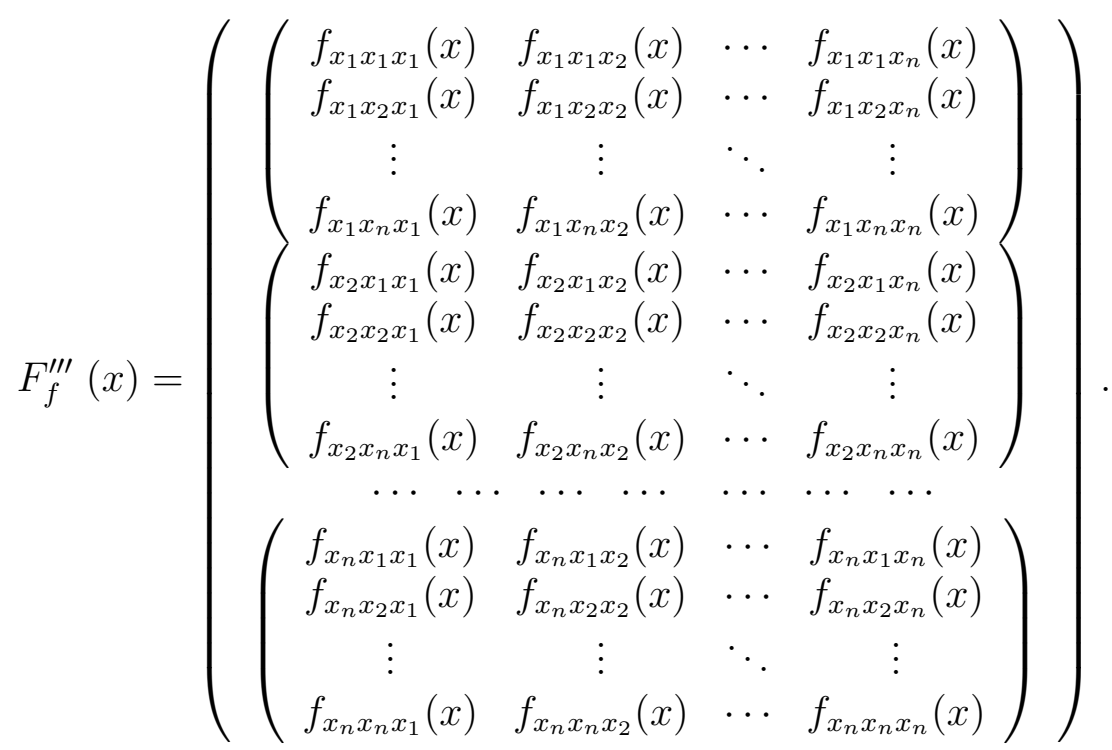

Taking into account (2), (3), the last formula can be expressed as following

$$
F_{f}^{\prime \prime \prime}(x)=\left(S_{f, 1}^{(3)}(x) S_{f, 2}^{(3)}(x) \ldots S_{f, n}^{(3)}(x)\right)^{T} .
$$

If $k=3$, formula (6) becomes (4). 
Assume that for $k=k_{0},\left(k_{0}>3\right)$, the conclusions of the theorem are valid. Let us show, that for $k=k_{0}+1$ the theorem is also true. According to the assumption, the mapping $F_{f}^{\left(k_{0}\right)}(x)$ of the space $K(\underbrace{\mathbb{R}^{n} \times \mathbb{R}^{n} \times \cdots \times \mathbb{R}^{n}}_{k_{0} \text { times }}, \mathbb{R}^{m})$ has components $F_{f_{x_{1}}}^{\left(k_{0}\right)}(x)$, $F_{f_{x_{2}}}^{\left(k_{0}\right)}(x), \ldots, F_{f_{x_{n}}}^{\left(k_{0}\right)}(x)$, that is,

$$
F_{f}^{\left(k_{0}\right)}(x)=\left(F_{f_{x_{1}}}^{\left(k_{0}\right)}(x) F_{f_{x_{2}}}^{\left(k_{0}\right)}(x) \ldots F_{f_{x_{n}}}^{\left(k_{0}\right)}(x)\right)^{T} .
$$

Since $F_{f}^{\left(k_{0}+1\right)}(x)=\left(F_{f}^{\left(k_{0}\right)}(x)\right)^{\prime}$, by Lemma 1 we obtain

$$
F_{f}^{\left(k_{0}+1\right)}(x)=\left(\left(F_{f_{x_{1}}}^{\left(k_{0}\right)}(x)\right)^{\prime}\left(F_{f_{x_{2}}}^{\left(k_{0}\right)}(x)\right)^{\prime} \ldots\left(F_{f_{x_{n}}}^{\left(k_{0}\right)}(x)\right)^{\prime}\right)^{T} .
$$

But the components $F_{f_{x_{i}}}^{\left(k_{0}\right)}(x),(i=\overline{1, n})$ consist of $n$ components $F_{f_{x_{i} x_{j}}}^{\left(k_{0}\right)}(x),(j=\overline{1, n})$. So by Lemma 1 it follows from (7) that

$$
F_{f}^{\left(k_{0}+1\right)}(x)=\left(C_{1} C_{2} \ldots C_{n}\right)^{T}, \quad C_{j}=\left(\left(F_{f_{x_{j} x_{1}}}^{\left(k_{0}\right)}(x)\right)^{\prime}\left(F_{f_{x_{j} x_{2}}}^{\left(k_{0}\right)}(x)\right)^{\prime} \ldots\left(F_{f_{x_{j} x_{n}}}^{\left(k_{0}\right)}(x)\right)^{\prime}\right)^{T}
$$

$(1 \leq j \leq n)$ and so on, until the mapping components $F_{f}^{\left(k_{0}\right)}(x)$ become the generating matrices $S_{f, i}^{\left(k_{0}\right)}(x),\left(i=\overline{1, n^{k_{0}-2}}\right)$. The determination of the derivative $F_{f}^{\left(k_{0}+1\right)}(x)$ is reduced to the finding the first derivative of mapping, components of which are generating matrices of derivative $F_{f}^{\left(k_{0}\right)}(x)$. Now the derivatives of these mappings are found successively to each such bilinear mapping. As a result, we are convinced that all conclusions of the theorem are true statements. Thus, this theorem is proved.

Remark 2. It follows from the proved theorem that the orders of differentiation with respect to variables $x_{1}, x_{2}, \ldots, x_{n}$ in each functional $f_{x_{(2)} x_{(3)} \ldots x_{(k)}}(x)$ are different, and they together cover the whole set of possible sequences of differentiations, each of which is performed $k$ times.

From Theorem 2, we obtain the following algorithm to find derivative $F_{f}^{(k)}(x)$ of functional $f$ and some of its generating matrices $S_{f, i}^{(k)}(x)$.

The algorithm for determination of derivative $F_{f}^{(k)}(x)$ of functional $f$ and its generating matrices $S_{f, i}^{(k)}(x)$ has been developed using Theorem 2 .

Algorithm 1. If the functional $f: \mathbb{R}^{n} \rightarrow \mathbb{R}^{1}$ has the derivative of the $k$-th order, $(k>2)$, then it is a hypermatrix $F_{f}^{(k)}(x)((k-1)$-linearly ordered set of all generating matrices $\left.S_{f, i}^{(k)}(x),\left(i=\overline{1, N}, N=n^{k-2}\right)\right)$, in which the variables of differentiating in the functionals $f_{x_{(2)} x_{(3)} \ldots x_{(k)}}(x)$ of generating matrices are as follows:

1. differentiation with respect to $x_{(2)}$ of all functionals of generating matrices

- whose numbers belong to the interval $\left[1, N_{1}\right]$ where $N_{1}=N / n$ is the differentiation with respect to $x_{1}$,i.e, $x_{(2)} \equiv x_{1}$;

- whose numbers belong to the interval $\left[N_{1}+1,2 N_{1}\right]$, is the differentiation with respect to $x_{2}$, i.e., $x_{(2)} \equiv x_{2}$; 
- and so on;

- whose numbers belong to the interval $\left[(n-1) N_{1}+1, N\right]$, is the differentiation with respect to $x_{n}$, i.e., $x_{(2)} \equiv x_{n}$;

2. differentiation with respect to $x_{(3)}$ of all functionals of generating matrices

- whose numbers belong to the intervals $\left[1, N_{2}\right],\left[N_{1}+1, N_{1}+N_{2}\right],\left[2 N_{1}+1,2 N_{1}+\right.$ $\left.N_{2}\right] \ldots,\left[(n-1) N_{1}+1,(n-1) N_{1}+N_{2}\right]$, where $N_{2}=N_{1} / n$, is the differentiation with respect to $x_{1}$, i.e., $x_{(3)} \equiv x_{1}$;

- whose numbers belong to the intervals $\left[N_{2}+1,2 N_{2}\right],\left[N_{1}+N_{2}+1, N_{1}+2 N_{2}\right]$, $\left[2 N_{1}+1,2 N_{1}+N_{2}\right] \ldots,\left[(n-1) N_{1}+1,(n-1) N_{1}+N_{2}\right]$, is the differentiation with respect to $x_{2}$, i.e. $x_{(2)} \equiv x_{2}$;

- and so on;

- whose numbers belong to the intervals $\left[(n-1) N_{2}+1, N_{1}\right],\left[N_{1}+(n-1) N_{2}+1,2 N_{1}\right]$, $\left[2 N_{1}+(n-1) N_{2}+1,3 N_{1}\right], \ldots,\left[(n-1) N_{1}+(n-1) N_{2}+1, N\right]$, is the differentiation with respect to $x_{n}$, i.e., $x_{(3)} \equiv x_{n}$;

- and so on;

- as long as the differentiation is performed with respect to $x_{(k-1)}$;

$k-1$. Differentiation with respect to $x_{(k-1)}$ of all functionals of generating matrices

- in all the first rows is the differentiation with respect to $x_{1}$,

- in all the second rows is the differentiation with respect to $x_{2}$,

- and so on.

- in all $n$-th rows is the differentiation with respect to $x_{n}$,

$k$. Differentiation with respect to $x_{(k-1)}$ of all functionals of generating matrices

- in all the first rows is the differentiation with respect to $x_{1}$,

- in all the second rows is the differentiation with respect to $x_{2}$,

- and so on.

- in all $n$-th rows is the differentiation with respect to $x_{n}$.

Example 1. Let the function $f(x, y, z)$ be four times differentiable. Calculate its derivatives up to the four orders inclusively.

To simplify the notations, in the functions $f_{x_{(2)} x_{(3)} \ldots x_{(k)}}(x)$ we will not specify the value of argument $x$, remembering that this value in all the functions is the same. Then, successively applying at $n=3$ Formula (5) and Algorithm 1, we obtain

$$
\begin{aligned}
& f^{\prime}(x, y, z)=\left(f_{x}, f_{y}, f_{z}\right), \quad f^{\prime \prime}(x, y, z)=\left(\begin{array}{ccc}
f_{x x} & f_{x y} & f_{x z} \\
f_{y x} & f_{y y} & f_{y z} \\
f_{z x} & f_{z y} & f_{z z}
\end{array}\right) \\
& f^{\prime \prime \prime}(x, y, z)=\left(\left(\begin{array}{ccc}
f_{x x x} & f_{x x y} & f_{x x z} \\
f_{x y x} & f_{x y y} & f_{x y z} \\
f_{x z x} & f_{x z y} & f_{x z z}
\end{array}\right)\left(\begin{array}{lll}
f_{y x x} & f_{y x y} & f_{y x z} \\
f_{y y x} & f_{y y y} & f_{y y z} \\
f_{y z x} & f_{y z y} & f_{y z z}
\end{array}\right)\left(\begin{array}{lll}
f_{z x x} & f_{z x y} & f_{z x z} \\
f_{z y x} & f_{z y y} & f_{z y z} \\
f_{z z x} & f_{z z y} & f_{z z z}
\end{array}\right)\right)^{T}, \\
& f^{(4)}(x, y, z)=\left(\begin{array}{lll}
A_{1} & A_{2} & A_{3}
\end{array}\right)^{T}, A_{j}=\left(\begin{array}{lll}
B_{j 1} & B_{j 2} & B_{j 3}
\end{array}\right)^{T}, \\
& B_{11}=\left(\begin{array}{lll}
f_{x x x x} & f_{x x x y} & f_{x x x z} \\
f_{x x y x} & f_{x x y y} & f_{x x y z} \\
f_{x x z x} & f_{x x z y} & f_{x x z z}
\end{array}\right), B_{12}=\left(\begin{array}{lll}
f_{x y x x} & f_{x y x y} & f_{x y x z} \\
f_{x y y x} & f_{x y y y} & f_{x y y z} \\
f_{x y z x} & f_{x y z y} & f_{x y z z}
\end{array}\right), B_{13}=\left(\begin{array}{lll}
f_{x z x x} & f_{x z x y} & f_{x z x z} \\
f_{x z y x} & f_{x z y y} & f_{x z y z} \\
f_{x z z x} & f_{x z z y} & f_{x z z z}
\end{array}\right),
\end{aligned}
$$


$B_{21}=\left(\begin{array}{lll}f_{y x x x} & f_{y x x y} & f_{y x x z} \\ f_{y x y x} & f_{y x y y} & f_{y x y z} \\ f_{y x z x} & f_{y x z y} & f_{y x z z}\end{array}\right), B_{22}=\left(\begin{array}{lll}f_{y y x x} & f_{y y x y} & f_{y y x z} \\ f_{y y y x} & f_{y y y y} & f_{y y y z} \\ f_{y y z x} & f_{y y z y} & f_{y y z z}\end{array}\right), B_{23}=\left(\begin{array}{lll}f_{y z x x} & f_{y z x y} & f_{y z x z} \\ f_{y z y x} & f_{y z y y} & f_{y z y z} \\ f_{y z z x} & f_{y z z y} & f_{y z z z}\end{array}\right)$,
$B_{31}=\left(\begin{array}{llll}f_{z x x x} & f_{z x x y} & f_{z x x z} \\ f_{z x y x} & f_{z x y y} & f_{z x y z} \\ f_{z x z x} & f_{z x z y} & f_{z x z z}\end{array}\right), B_{32}=\left(\begin{array}{lll}f_{z y x x} & f_{z y x y} & f_{z y x z} \\ f_{z y y x} & f_{z y y y} & f_{z y y z} \\ f_{z y z x} & f_{z y z y} & f_{z y z z}\end{array}\right), B_{33}=\left(\begin{array}{lll}f_{z z x x} & f_{z z x y} & f_{z z x z} \\ f_{z z y x} & f_{z z y y} & f_{z z y z} \\ f_{z z z x} & f_{z z z y} & f_{z z z z}\end{array}\right)$.

Remark 3. If the functional $f: \mathbb{R}^{n} \rightarrow \mathbb{R}^{1}$ has all continuous partial derivatives of the $k$-th order, $(k>2)$, then all the generating matrixes $S_{f, i}^{(k)}(x),\left(i=\overline{1, N}, N=n^{k-2}\right)$ are the symmetric ones.

Since the derivative of the functional vector is a vector of derivatives of these functionals, then from Theorem 2 we have the following corollary.

Corollary 2. Let the mappings $F: \mathbb{R}^{n} \rightarrow \mathbb{R}^{m}(m>1)$, whose components $f_{i}: \mathbb{R}^{n} \rightarrow \mathbb{R}^{1}$ $(m>1)$ are functionals, be differentiable. Then a linearly structured hypermatrix

$$
F^{\prime}(x)=\left(f_{1}^{\prime}(x) f_{2}^{\prime}(x) \ldots f_{m}^{\prime}(x)\right)^{T}
$$

is a derivative of this mapping at the point $x$, where $f_{i}^{\prime}(x)$ are the derivatives of the corresponding components $f_{i}(x)$ of mapping $F$.

\section{Matrix representation of Taylor's formula for mappings in finite-dimensio-} nal spaces. Let the mapping $f: D \subset \mathbb{R}^{n} \rightarrow \mathbb{R}^{1}$ be given. Thus, $f(x)=f\left(x_{1}, x_{2}, \ldots, x_{n}\right)$. Assume that in the neighborhood $D_{0} \subset D$ of a certain point $x=\left(x_{1}, x_{2}, \ldots, x_{n}\right)$ this function has all continuous partial derivatives up to $k+1$ order, inclusively. We will give the argument such an increase $\Delta$ that the point $x+\Delta$ also belongs to the neighborhood $D_{0}$ of the point $x$. Then by Taylor's formula

$$
\begin{aligned}
f(x+\Delta) & =f(x)+\frac{1}{1 !} \sum_{i=1}^{n} \frac{\partial f(x)}{\partial x_{i}} \Delta_{i}+\frac{1}{2 !} \sum_{i=1}^{n} \sum_{j=1}^{n} \frac{\partial^{2} f(x)}{\partial x_{i} \partial x_{j}} \Delta_{i} \Delta_{j}+\cdots+ \\
& +\frac{1}{k !} \sum_{i_{1}=1}^{n} \sum_{i_{2}=1}^{n} \cdots \sum_{i_{k}=1}^{n} \frac{\partial^{k} f(x)}{\partial x_{i_{1}} \partial x_{i_{2}} \cdots \partial x_{i_{k}}} \Delta_{i_{1}} \Delta_{i_{2}} \cdots \Delta_{i_{k}} \\
& +\frac{1}{(k+1) !} \sum_{i_{1}=1}^{n} \sum_{i_{2}=1}^{n} \cdots \sum_{i_{k+1}=1}^{n} \frac{\partial^{k+1} f(x+\theta \Delta)}{\partial x_{i_{1}} \partial x_{i_{2}} \cdots \partial x_{i_{k+1}}} \Delta_{i_{1}} \Delta_{i_{2}} \cdots \Delta_{i_{k+1}}
\end{aligned}
$$

where $\Delta=\left(\Delta_{1}, \Delta_{2}, \cdots, \Delta_{n}\right)^{T}$ or using the total differentials,

$$
\begin{gathered}
f(x+\Delta)=f(x)+\frac{1}{1 !} d f(x)+\frac{1}{2 !} d^{2} f(x)+\cdots+\frac{1}{k !} d^{k} f(x)+\frac{1}{(k+1) !} d^{k+1} f(x+\theta \cdot \Delta)= \\
f(x)+\frac{1}{1 !}\left(\sum_{i=1}^{n} \frac{\partial}{\partial x_{i}} \cdot \Delta_{i}\right)^{[1]} f(x)+\frac{1}{2 !}\left(\sum_{i=1}^{n} \frac{\partial}{\partial x_{i}} \cdot \Delta_{i}\right)^{[2]} f(x)+\frac{1}{k !}\left(\sum_{i=1}^{n} \frac{\partial}{\partial x_{i}} \cdot \Delta_{i}\right)^{[k]} f(x)+ \\
+\frac{1}{(k+1) !}\left(\sum_{i=1}^{n} \frac{\partial}{\partial x_{i}} \cdot \Delta_{i}\right)^{[k+1]} f(x+\theta \cdot \Delta)
\end{gathered}
$$


where the following symbolic notation is used

$$
\begin{gathered}
d^{s} f(x)=\left(\frac{\partial}{\partial x_{1}} \Delta_{1}+\frac{\partial}{\partial x_{2}} \Delta_{2}+\cdots+\frac{\partial}{\partial x_{n}} \Delta_{n}\right)^{[s]} \cdot f(x)=\left(\sum_{i=1}^{n} \frac{\partial}{\partial x_{i}} \Delta_{i}\right)^{[s]} \cdot f(x) \\
(s=\overline{1, k+1}) .
\end{gathered}
$$

The conclusions of the following theorem make possible to show the process of computing differentials of the functional by means of operations over the generating matrices of its corresponding derivatives.

Theorem 3. Let the functional $f: D \subset \mathbb{R}^{n} \rightarrow \mathbb{R}^{1}$ be $k$ times continuously differentiable in the neighborhood $D_{0} \subset D$ of a certain point $x$. Then its differential of order $k$ at this point is

$$
\begin{gathered}
d^{k} f(x)=\Delta^{T}\left(\cdots \left(\Delta ^ { T } \left(\Delta^{T}\left(\Delta^{T} S_{f, 1}^{(k)} \Delta, \Delta^{T} S_{f, 2}^{(k)} \Delta, \cdots, \Delta^{T} S_{f, n}^{(k)} \Delta\right)^{T},\right.\right.\right. \\
\Delta^{T}\left(\Delta^{T} S_{f, n+1}^{(k)} \Delta, \Delta^{T} S_{f, n+2}^{(k)} \Delta, \cdots, \Delta^{T} S_{f, 2 n}^{(k)} \Delta\right)^{T}, \cdots, \\
\left.\Delta^{T}\left(\Delta^{T} S_{f,(n-1) n+1}^{(k)} \Delta, \Delta^{T} S_{f,(n-1) n+2}^{(k)} \Delta, \cdots, \Delta^{T} S_{f, n^{2}}^{(k)} \Delta\right)^{T}\right)^{T}, \cdots, \\
\left(\Delta ^ { T } \left(\Delta ^ { T } \left(\Delta^{T} S_{f,\left(n^{k-4}-1\right) n+1}^{(k)} \Delta, \Delta^{T} S_{f,\left(n^{k-4}-1\right) n+2}^{(k)} \Delta, \cdots, \Delta^{T} S_{f, n^{k-4}}^{(k)} \Delta^{T}, \cdots,\right.\right.\right. \\
\left.\left.\Delta^{T}\left(\Delta^{T} S_{f,\left(n^{k-3}-1\right) n+1}^{(k)} \Delta, \Delta^{T} S_{f,\left(n^{k-3}-1\right) n+2}^{(k)} \Delta, \cdots, \Delta^{T} S_{f, n^{k-2}}^{(k)} \Delta\right)^{T}\right)^{T} \cdots\right)^{T},
\end{gathered}
$$

Proof. From Algorithm 1 of obtaining a derivative of the functional, it follows that the hypermatrix (4) contains all its partial derivatives. We directly convince that for all such $x, \Delta \in \mathbb{R}^{n}$ that $x+\Delta \in D_{0}$, the quantity $f^{\prime}(x) \Delta$ is a total differential of the first order of the function $f(x)$, and $\Delta^{T} f^{\prime \prime}(x) \Delta$ is its second-order differential.

Let $k=3$. Then, we successively get

$$
\left.\begin{array}{c}
d^{3} f(x)=d\left(d^{2} f(x)\right)=d\left(\Delta^{T} S_{f, 1}^{(2)}(x) \Delta\right)=d\left(\Delta^{T} f^{\prime \prime}(x) \Delta\right)=\Delta^{T} d\left(f^{\prime \prime}(x) \Delta\right)= \\
=\Delta^{T} d\left(\left(\begin{array}{c}
\left(f_{x_{1}}\right)^{\prime} \Delta \\
\left(f_{x_{2}}\right)^{\prime} \Delta \\
\vdots \\
\left(f_{x_{n}}\right)^{\prime} \Delta
\end{array}\right)=\Delta^{T}\left(\begin{array}{c}
d\left(\left(f_{x_{1}}\right)^{\prime} \Delta\right) \\
d\left(\left(f_{x_{2}}\right)^{\prime} \Delta\right) \\
\vdots \\
d\left(\left(f_{x_{n}}\right)^{\prime} \Delta\right)
\end{array}\right)=\Delta^{T}\left(\begin{array}{c}
\left.\Delta^{T} d\left(\left(f_{x_{1}}\right)^{\prime}\right)^{T}\right) \\
\Delta^{T} d\left(\left(\left(f_{x_{2}}\right)^{\prime}\right)^{T}\right) \\
\vdots \\
\Delta^{T}\left(\begin{array}{c}
d f_{x_{1} x_{1}} \\
\vdots \\
d f_{x_{1} x_{n}}
\end{array}\right) \\
\vdots \\
d f_{x_{n} x_{1}} \\
\vdots \\
d f_{x_{n} x_{n}}
\end{array}\right)\right.
\end{array}\right)=
$$




$$
=\Delta^{T}\left(\begin{array}{c}
\Delta^{T} S_{f, 1}^{(3)} \Delta \\
\vdots \\
\Delta^{T} S_{f, n}^{(3)} \Delta
\end{array}\right)=\Delta^{T}\left(\Delta^{T} S_{f, 1}^{(3)} \Delta, \cdots, \Delta^{T} S_{f, n}^{(3)} \Delta\right)^{T} .
$$

Let formula (12) be valid for $k=k_{0}$. Let us prove that it is valid for $k=k_{0}+1$. Indeed, in this case

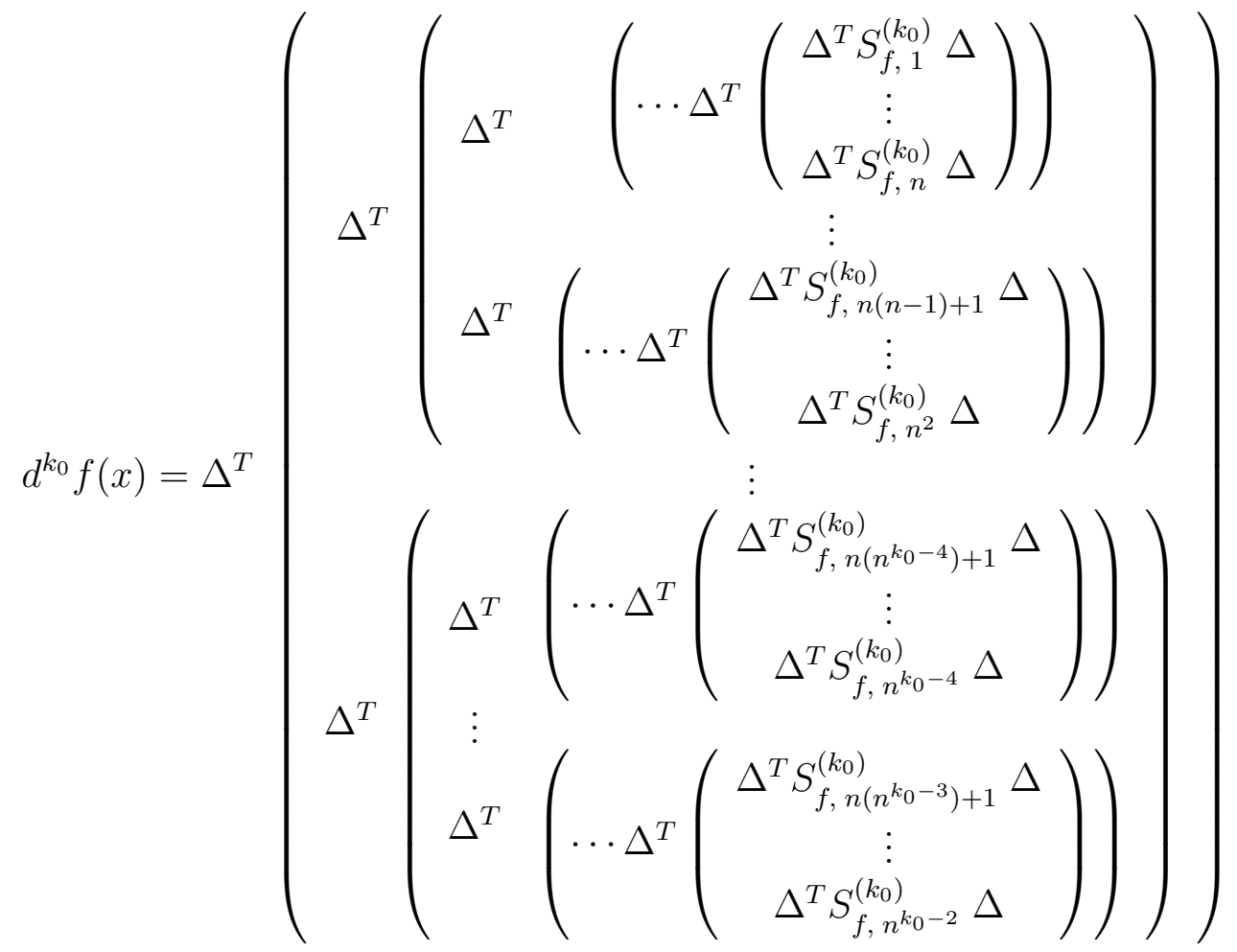

Since $d^{k_{0}+1} f(x)=d\left(d^{k_{0}} f(x)\right)$, one has

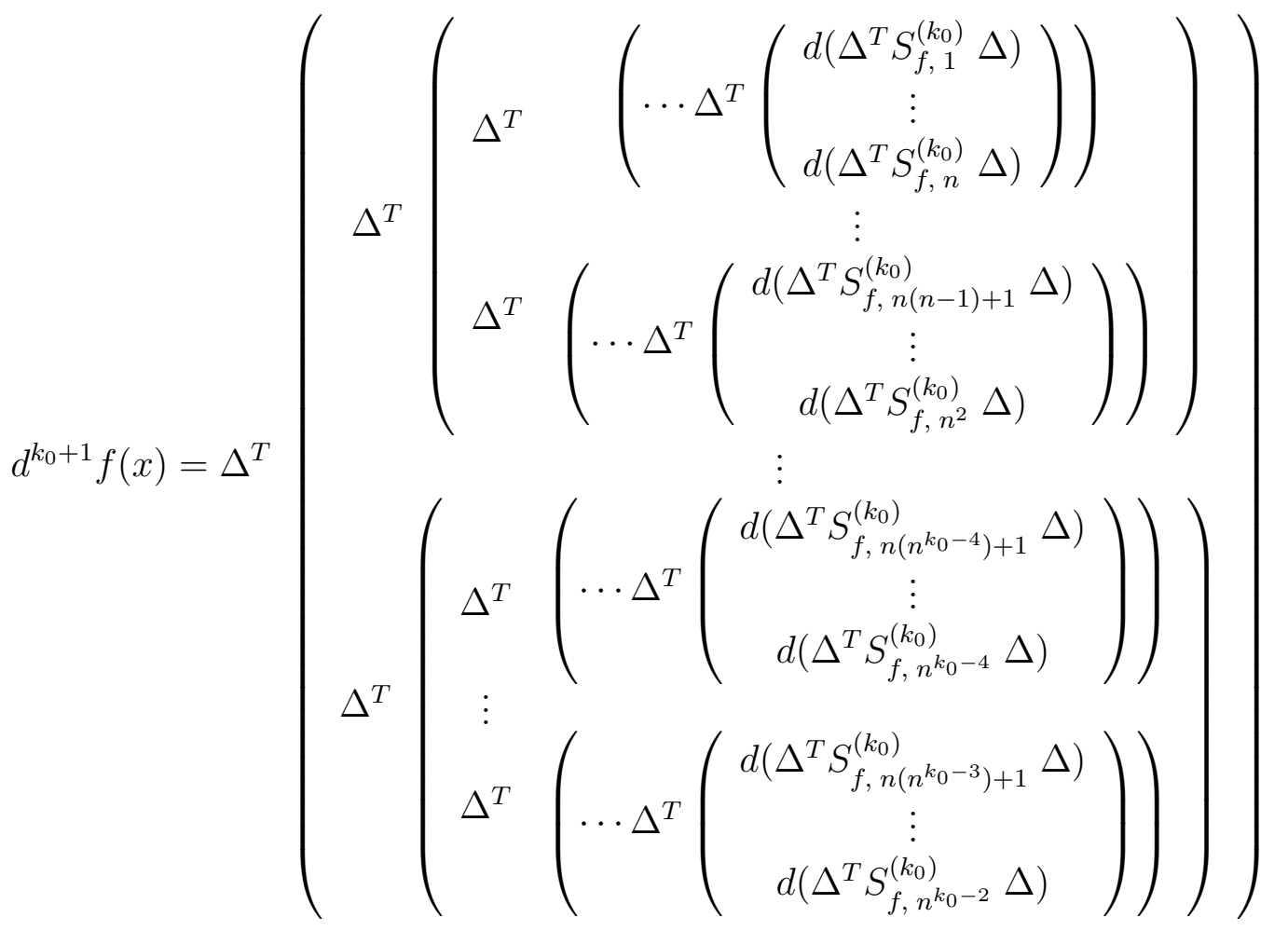


Therefore, it is sufficient to prove the validity of the statement for any single component of this mapping. For example, let us prove it for its first component.

$$
\begin{aligned}
& d\left(\Delta^{T} S_{f, 1}^{\left(k_{0}\right)}(x) \Delta\right)=\Delta^{T} d\left(S_{f, 1}^{\left(k_{0}\right)}(x) \Delta\right)= \\
& =\Delta^{T} d\left(\left(\begin{array}{c}
\left(f_{x_{1} x_{1} \cdots x_{1} x_{1}}\right)^{\prime} \Delta \\
\left(f_{x_{1} x_{1} \cdots x_{1} x_{2}}\right)^{\prime} \Delta \\
\vdots \\
\left(f_{x_{1} x_{1} \cdots x_{1} x_{n}}\right)^{\prime} \Delta
\end{array}\right)=\Delta^{T}\left(\begin{array}{c}
d\left(\left(f_{x_{1} x_{1} \cdots x_{1} x_{1}}\right)^{\prime} \Delta\right) \\
d\left(\left(f_{x_{1} x_{1} \cdots x_{1} x_{2}}\right)^{\prime} \Delta\right) \\
\vdots \\
d\left(\left(f_{x_{1} x_{1} \cdots x_{1} x_{n}}\right)^{\prime} \Delta\right)
\end{array}\right)=\right. \\
& =\Delta^{T}\left(\begin{array}{c}
\Delta^{T} d\left(\left(\left(f_{x_{1} x_{1} \cdots x_{1} x_{1}}\right)^{\prime}\right)^{T}\right) \\
\Delta^{T} d\left(\left(\left(f_{x_{1} x_{1} \cdots x_{1} x_{2}}\right)^{\prime}\right)^{T}\right) \\
\vdots \\
\Delta^{T} d\left(\left(\left(f_{x_{1} x_{1} \cdots x_{1} x_{n}}\right)^{\prime}\right)^{T}\right)
\end{array}\right)=\Delta^{T}\left(\begin{array}{c}
\Delta^{T}\left(\begin{array}{c}
d f_{x_{1} x_{1} \cdots x_{1} x_{1} x_{1}} \\
\vdots \\
d f_{x_{1} x_{1} \cdots x_{1} x_{1} x_{n}}
\end{array}\right) \\
\vdots \\
\Delta^{T}\left(\begin{array}{c}
d f_{x_{1} x_{1} \cdots x_{1} x_{n} x_{1}} \\
\vdots \\
d f_{x_{1} x_{1} \cdots x_{1} x_{n} x_{n}}
\end{array}\right)
\end{array}\right)=
\end{aligned}
$$

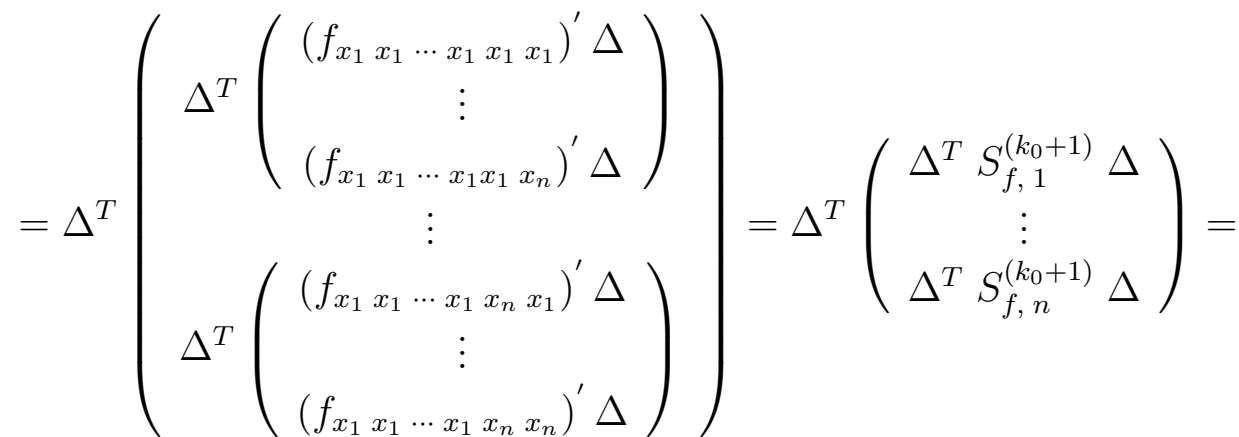

$$
\begin{aligned}
& =\Delta^{T}\left(\Delta^{T} S_{f, 1}^{\left(k_{0}+1\right)} \Delta, \cdots, \Delta^{T} S_{f, n}^{\left(k_{0}+1\right)} \Delta\right)^{T} .
\end{aligned}
$$

Taking into account Remark 2, we convince that $d^{k_{0}+1} f(x)$ is the total $k$-th order differential of the functional $f$. The theorem is proved.

From (10), (11) we deduce the following corollary.

Corollary 3. Let a mapping $F: \mathbb{R}^{n} \rightarrow \mathbb{R}^{m},(m>1)$, whose components are the functionals $f_{i}: \mathbb{R}^{n} \rightarrow \mathbb{R}^{1},(i=\overline{1, m})$, be $k$ times differentiable. Then the vector

$$
d^{k} F(x)=\left(d^{k} f_{1}(x), d^{k} f_{2}(x), \cdots, d^{k} f_{m}(x)\right)^{T},
$$

where $d^{k} f_{i}(x)$ are differentials of $k$-th order of the corresponding components $f_{i}(x)$ of mappi$n g F$, is $k$-th order differential of $F$ at the point $x$.

Example 2. Let a function $f(x, y, z)$ be four times differentiable. Construct its differentials of the fourth orders in a matrix form.

To simplify the notations, let us do not specify the argument value $x$ in the functions $f_{x_{(2)} x_{(3)} \ldots x_{(k)}}(x)$ and let us assume this argument is the same in all functions. Then, successively applying formula (12), the following results are obtained

$$
d^{(4)} f(x, y, z)=
$$




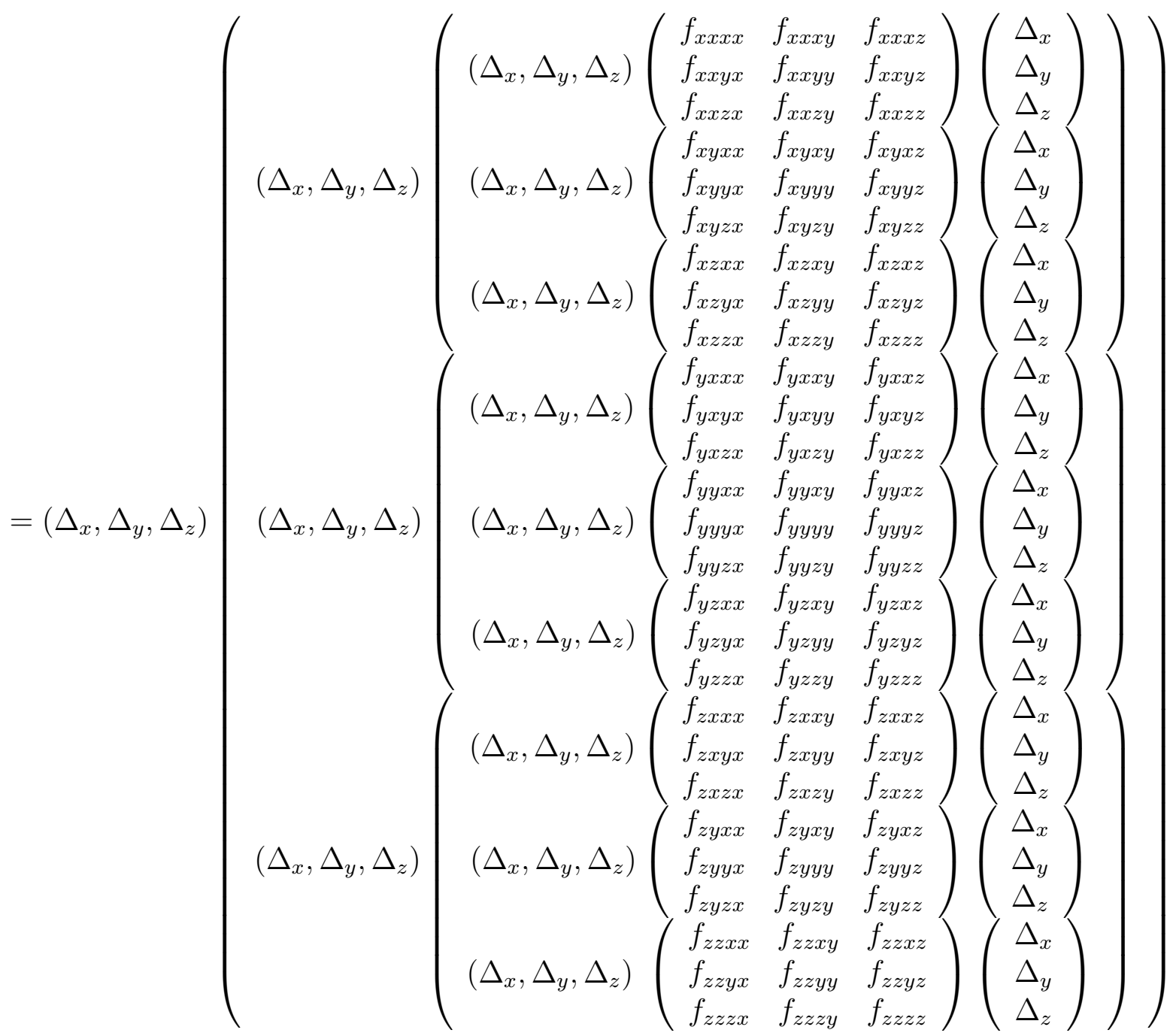

From Theorems 2, 3, Corollaries 2, 3 and equalities (10), (11) the following corollary is obtained.

Corollary 4. Let the mapping $F: D \subset \mathbb{R}^{n} \rightarrow \mathbb{R}^{m},(m>1)$, whose components are the functionals $f_{i}: \mathbb{R}^{n} \rightarrow \mathbb{R}^{1},(i=\overline{1, m})$, be $k$-th times continuously differentiable in the neighborhood $D_{0} \subset D$ of the point $x$. If $x+\Delta \in D_{0}$, then

$$
\begin{gathered}
F(x+\Delta)=F(x)+F^{\prime}(x) \cdot \Delta+\frac{1}{2 !} F^{\prime \prime}(x) \cdot(\Delta, \Delta)+\cdots+ \\
+\frac{1}{(k-1) !} F^{(k-1)}(x) \cdot(\Delta)^{k-1}+\frac{1}{k !} F^{(k)}(x+\{\theta\} \Delta) \cdot(\Delta)^{k},
\end{gathered}
$$

where $(\Delta)^{n}=\underbrace{(\Delta, \cdots, \Delta)}_{n \text { times }}, \Delta=\left(\Delta_{1}, \Delta_{2}, \cdots, \Delta_{n}\right)^{T}, 0<\theta<1$,

$$
\begin{gathered}
F(x+\Delta)=F(x)+d^{1} F(x)+\frac{1}{2 !} d^{2} F(x)+\cdots+ \\
+\frac{1}{(k-1) !} d^{k-1} F(x)+\frac{1}{k !} d^{k} F(x+\{\theta\} \Delta) .
\end{gathered}
$$

The derivatives $F^{(j)}(x)$ and differentials $d^{j} F(x),(j=\overline{1, k})$ for $(14),(15)$ can be found in accordance with (9), (13), respectively. The intermediate points $x+\theta_{i} \Delta,\left(0<\theta_{i}<1\right)$ 
in the components $f_{i}^{(k)}\left(x+\theta_{i} \Delta\right), d^{k} f_{i}\left(x+\theta_{i} \Delta\right)$, the mapping $F^{(k)}(x+\{\theta\} \Delta)$ and vector $d^{k} F(x+\{\theta\} \Delta)$, generally speaking, are different, so the mapping $F^{(k)}(x+\{\theta\} \Delta)$ and vector $d^{k} F(x+\{\theta\} \Delta)$ can not be $k$-th Gateaux derivative and $j$-th order differential of mapping $F$ respectively.

Formulas (14), (15) are a matrix representation of the Taylor formula for mappings.

4. Methods for eliminating functional uncertainties when the expansion interval of the mapping shrinks to a point. The conclusion of the following theorem makes possible to analyze the local uncertainties in the residual terms of the Taylor formula and to construct and explore new effective methods [3-5] for solving systems of nonlinear algebraic equations.

Theorem 4. Let the mapping $F: D \subset \mathbb{R}^{n} \rightarrow \mathbb{R}^{m}$ be $(k+1)$ times continuously differentiable by Fréchet in the neighborhood $D_{0} \subset D$ of a certain point $x$, all $(k+1)$-th order partial derivatives of all its components $f_{i},(i=\overline{1, n})$ are not identically equal to zero and at least one of the equalities $f_{i}(0)=0, f_{i}^{\prime}(0)=0, \cdots, f_{i}^{(k-1)}(0)=0$ is not fulfilled. If $x+\Delta \in D_{0}$, then

$$
\lim _{\Delta \rightarrow 0} \theta^{(k)}=\frac{1}{k+1}
$$

where $x+\theta^{(k)} \Delta,\left(0<\theta^{(k)}<1\right)$ is the intermediate point in the remainder term in formula (10).

Proof. Let the conditions of the theorem be satisfied. Firstly, we prove that the value $\theta^{(k)}$ as a function of $\Delta$, is not a constant value. In fact, if $f_{i}(x)=0, f_{i}^{\prime}(x)=0, \cdots, f_{i}^{(k-1)}(x)=0$ but all other conditions of the theorem are fulfilled, then

$$
f_{m}(x+\Delta)=\frac{1}{k !} \sum_{i_{1}=1}^{n} \sum_{i_{2}=1}^{n} \cdots \sum_{i_{k}=1}^{n} \frac{\partial^{k} f_{m}\left(x+\theta_{m}^{(k)} \Delta\right)}{\partial x_{i_{1}} \partial x_{i_{2}} \cdots \partial x_{i_{k}}} \Delta_{i_{1}} \Delta_{i_{2}} \cdots \Delta_{i_{k}} .
$$

All terms in the right-hand side of this equality contain products of the form $\Delta_{i_{1}} \Delta_{i_{2}} \cdots \Delta_{i_{k}}$ and are not identical to certain constants. Therefore, the functions $f_{m}(x+\Delta)=u_{m}(x+$ $\Delta) \Delta_{i_{1}}^{s_{1}} \Delta_{i_{2}}^{s_{2}} \cdots \Delta_{k}^{s_{k}},\left(s_{j} \geq 1, j=\overline{1, k}\right)$. Since $\Delta \neq 0$, we have $x=0$.

Therefore,

$$
u_{m}(\Delta) \Delta_{i_{1}}^{s_{1}} \Delta_{i_{2}}^{s_{2}} \cdots \Delta_{i_{k}}^{s_{k}}=\frac{1}{k !} \sum_{i_{1}=1}^{n} \sum_{i_{2}=1}^{n} \cdots \sum_{i_{k}=1}^{n} \frac{\partial^{k} f_{m}\left(\theta_{m}^{(k)} \Delta\right)}{\partial x_{i_{1}} \partial x_{i_{2}} \cdots \partial x_{i_{k}}} \Delta_{i_{1}} \Delta_{i_{2}} \cdots \Delta_{i_{k}} .
$$

Consequently, $\theta_{m}^{(k)}$ will not depend on $\Delta$ only when every term $\frac{\partial^{k} f_{m}\left(\theta_{m}^{(k)} \Delta\right)}{\partial x_{i_{1}} \partial x_{i_{2}} \cdots \partial x_{i_{k}}} \Delta_{i_{1}} \Delta_{i_{2}} \cdots \Delta_{i_{k}}$ has the following form $c_{j} g_{j}\left(\theta_{m}^{(k)}\right) u_{m}(\Delta) \Delta_{i_{1}}^{s_{1}} \Delta_{i_{2}}^{s_{2}} \cdots \Delta_{i_{k}}^{s_{k}}$, where $c_{j}$ are some non-zero constants. It follows that this is possible only for the functions $f_{m}(t)=c_{m} \Delta_{1}^{k_{1}} \Delta_{2}^{k_{2}} \cdots \Delta_{n}^{k_{n}}$, where $k_{j}=k+\alpha_{j}, \alpha_{j}>0$ are constants.

All differentials of each functional are the corresponding functionals, and all differentials of each mapping are linear mappings which components are also certain corresponding functionals. If the conditions of the theorem are satisfied then we have

$$
\begin{gathered}
F(x+\Delta)=F(x)+d^{1} F(x)+\frac{1}{2 !} d^{2} F(x)+\cdots+ \\
+\frac{1}{(k-1) !} d^{k-1} F(x)+\frac{1}{k !} d^{k} F\left(x+\left\{\theta^{(k)}\right\} \Delta\right) .
\end{gathered}
$$


Assume that the mapping $F$ has the components $f_{1}, f_{2}, \cdots, f_{m}$. Then, the mapping $d^{k} F\left(x+\left\{\theta^{(k)}\right\} \Delta\right)$ has the components $d^{k} f_{1}\left(x+\theta_{1}^{(k)} \Delta\right), d^{k} f_{2}\left(x+\theta_{2}^{(k)} \Delta\right), \cdots, \quad d^{k} f_{m}(x+$ $\left.\theta_{m}^{(k)} \Delta\right)$. Applying the mean value theorem to the mapping $d^{k} F\left(x+\left\{\theta^{(k)}\right\} \Delta\right)$, one consiquently obtains

$$
\begin{aligned}
& d^{k} F\left(x+\left\{\theta^{(k)}\right\} \Delta\right)=d^{k} F(x)+\left(d^{k} F\left(x+\left\{\theta^{(k)}\right\}\left\{\bar{\theta}^{(k)}\right\} \Delta\right)\right)^{\prime}\left\{\theta^{(k)}\right\} \Delta= \\
& =d^{k} F(x)+\left(\begin{array}{c}
\left(d^{k} f_{1}\left(x+\theta_{1}^{(k)} \bar{\theta}_{1}^{(k)} \Delta\right)\right)^{\prime} \\
\left(d^{k} f_{2}\left(x+\theta_{2}^{(k)} \bar{\theta}_{2}^{(k)} \Delta\right)\right)^{\prime} \\
\vdots \\
\left(d^{k} f_{m}\left(x+\theta_{m}^{(k)} \bar{\theta}_{m}^{(k)} \Delta\right)\right)^{\prime}
\end{array}\right)\left\{\theta^{(k)}\right\} \Delta= \\
& =d^{k} F(x)+\left(\begin{array}{c}
\left(d^{k} f_{1}\left(x+\theta_{1}^{(k)} \bar{\theta}_{1}^{(k)} \Delta\right)\right)^{\prime} \theta_{1}^{(k)} \Delta \\
\left(d^{k} f_{2}\left(x+\theta_{2}^{(k)} \bar{\theta}_{2}^{(k)} \Delta\right)\right)^{\prime} \theta_{2}^{(k)} \Delta \\
\vdots \\
\left(d^{k} f_{m}\left(x+\theta_{m}^{(k)} \bar{\theta}_{m}^{(k)} \Delta\right)\right)^{\prime} \theta_{m}^{(k)} \Delta
\end{array}\right)= \\
& =d^{k} F(x)+\left(\begin{array}{ccc}
\theta_{1}^{(k)} & d^{1}\left(d^{k} f_{1}\left(x+\theta_{1}^{(k)} \bar{\theta}_{1}^{(k)} \Delta\right)\right. \\
\theta_{2}^{(k)} & d^{1}\left(d^{k} f_{2}\left(x+\theta_{2}^{(k)} \bar{\theta}_{2}^{(k)} \Delta\right)\right. \\
\vdots & \\
\theta_{m}^{(k)} & d^{1}\left(d^{k} f_{m}\left(x+\theta_{m}^{(k)} \bar{\theta}_{m}^{(k)} \Delta\right)\right)
\end{array}\right) .
\end{aligned}
$$

Hence,

$$
d^{k} F\left(x+\left\{\theta^{(k)}\right\} \Delta\right)=d^{k} F(x)+\left\{\theta^{(k)}\right\} d^{k+1} F\left(x+\left\{\theta^{(k)}\right\}\left\{\bar{\theta}^{(k)}\right\} \Delta\right),
$$

where

$$
\{\theta\} d^{k+1} F\left(x+\left\{\theta_{1}\right\}\left\{\bar{\theta}_{1}\right\} \Delta\right)=\left(\begin{array}{ccc}
\theta_{1}^{(k)} & d^{(k+1)} f_{1}\left(x+\theta_{1}^{(k)}\right. & \left.\bar{\theta}_{1}^{(k)} \Delta\right) \\
\theta_{2}^{(k)} & d^{(k+1)} f_{2}\left(x+\theta_{2}^{(k)}\right. & \left.\bar{\theta}_{2}^{(k)} \Delta\right) \\
\vdots \\
\theta_{m}^{(k)} & d^{(k+1)} f_{m}\left(x+\theta_{m}^{(k)}\right. & \left.\bar{\theta}_{m}^{(k)} \Delta\right)
\end{array}\right) .
$$

Next, let us substitute (18) in (17). Then

$$
\begin{gathered}
F(x+\Delta)= \\
F(x)+d^{1} F(x)+\frac{1}{2 !} d^{2} F(x)+\cdots+\frac{1}{(k-1) !} d^{k-1} F(x)+ \\
\frac{1}{k !} d^{k} F(x)+\frac{1}{k !}\{\theta\} d^{k+1} F\left(x+\left\{\theta^{(k)}\right\}\left\{\bar{\theta}^{(k)}\right\} \Delta\right) .
\end{gathered}
$$

But under conditions of the theorem, the following equality is also fulfilled

$$
\begin{aligned}
F(x+\Delta)= & F(x)+d^{1} F(x)+\frac{1}{2 !} d^{2} F(x)+\cdots+\frac{1}{(k-1) !} d^{k-1} F(x)+ \\
& \frac{1}{k !} d^{k} F(x)+\frac{1}{(k+1) !} d^{k+1} F\left(x+\left\{\theta^{(k+1)}\right\} \Delta\right) .
\end{aligned}
$$


Hence,

$$
\frac{1}{k !}\{\theta\} d^{k+1} F\left(x+\left\{\theta^{(k)}\right\}\left\{\bar{\theta}^{(k)}\right\} \Delta\right)=\frac{1}{(k+1) !} d^{k+1} F\left(x+\left\{\theta^{(k+1)}\right\} \Delta\right),
$$

that is,

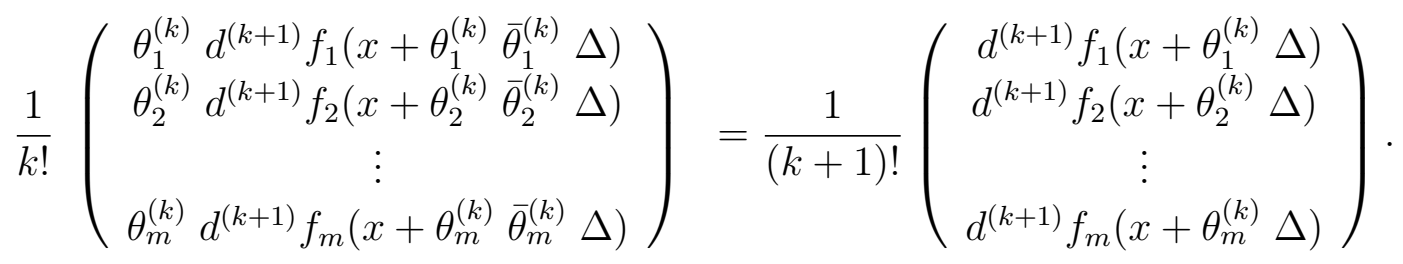

By Theorem 2 and Corollary 2, we state that equality (20) is equivalent to the equalities between all the corresponding generating matrices of each pair of the corresponding components of the mappings $\frac{1}{k !}\{\theta\} d^{k+1} F\left(x+\left\{\theta^{(k)}\right\}\left\{\bar{\theta}^{(k)}\right\} \Delta\right)$ and $\frac{1}{(k+1) !} d^{k+1} F\left(x+\left\{\theta^{(k+1)}\right\} \Delta\right)$. As elements of the generating matrices $S_{f_{i}, j}^{(k+1)}(x)\left(i=\overline{1, m} ; j=\overline{1, n^{k}}\right)$ are the corresponding functionals $g_{f_{i}, p q}^{(k+1)}(x),(p, q=\overline{1, n})$. Hence,

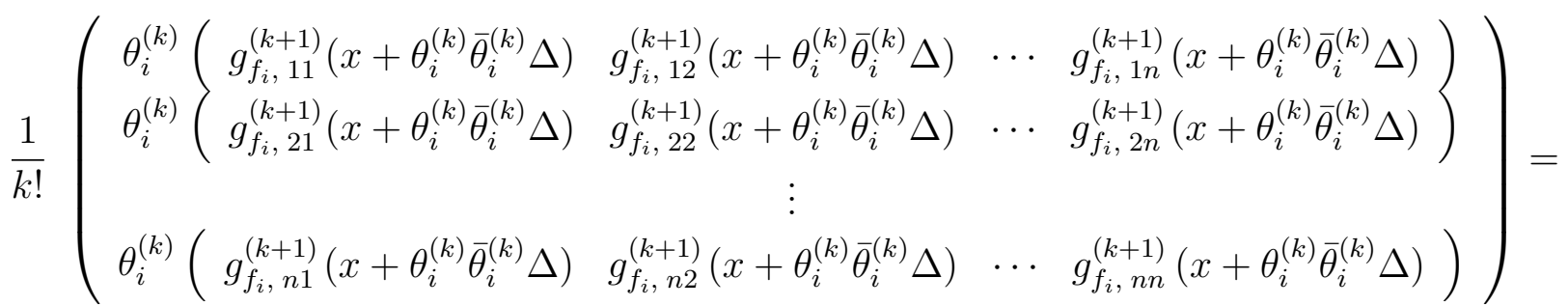

$$
\begin{aligned}
& =\frac{1}{(k+1) !}\left(\begin{array}{cccc}
g_{f_{i}, 11}^{(k+1)}\left(x+\theta_{i}^{(k+1)} \Delta\right) & g_{f_{i}, 12}^{(k+1)}\left(x+\theta_{i}^{(k+1)} \Delta\right) & \cdots & g_{f_{i}, 1 n}^{(k+1)}\left(x+\theta_{i}^{(k+1)} \Delta\right) \\
g_{f_{i}, 21}^{(k+1)}\left(x+\theta_{i}^{(k+1)} \Delta\right) & g_{f_{i}, 22}^{(k+1)}\left(x+\theta_{i}^{(k+1)} \Delta\right) & \cdots & g_{f_{i}, 2 n}^{(k+1)}\left(x+\theta_{i}^{(k+1)} \Delta\right) \\
\cdots & \cdots & \cdots & \cdots \\
g_{f_{i}, n 1}^{(k+1)}\left(x+\theta_{i}^{(k+1)} \Delta\right) & g_{f_{i}, n 2}^{(k+1)}\left(x+\theta_{i}^{(k+1)} \Delta\right) & \cdots & g_{f_{i}, n n}^{(k+1)}\left(x+\theta_{i}^{(k+1)} \Delta\right)
\end{array}\right) \text {, }
\end{aligned}
$$

where $i=\overline{1, m} ; \theta_{i}^{(k)}, \bar{\theta}_{i}^{(k)}, \theta_{i}^{(k+1)} \in(0,1)$.

Since, under the conditions of the theorem, all partial derivatives $g_{f_{i}, p q}^{(k+1)}(x),(p, q=\overline{1, n})$ in the neighborhood of point $x$, are continuous functions, then

$$
\lim _{\Delta \rightarrow 0} g_{f_{i}, p q}^{(k+1)}\left(x+\theta_{i}^{(k)} \Delta\right)=\lim _{\Delta \rightarrow 0} g_{f_{i}, p q}^{(k)}\left(x+\theta_{i}^{(k)} \bar{\theta}_{i}^{(k)} \Delta\right)=g_{f_{i}, p q}^{(k+1)}(x) .
$$

Thus, $\lim _{\Delta \rightarrow 0} \theta_{i}^{(k)}=\frac{1}{k+1}$ for all $i=\overline{1, m}$ that is equivalent to $(16)$.

\section{REFERENCES}

1. Kolmogorov A.N., Fomin S.V., Elements of the theory of functions and functional analysis. M.: Nauka, 1989. (in Russian)

2. Ortega J., Ranboldt V., Iterative methods for solving nonlinear systems of equations with many unknowns. M.: Mir, 1975. (in Russian)

3. Senio P.S., A new approach to the construction of interval methods for solving nonlinear equations, Visn. Lviv Univ., Ser. Mekh.-Mat., 31 (1989), 85-92 (in Ukrainian) 
4. Senio P.S., Interval methods for solving certain classes of deterministic problems, Visn. Lviv Univ., Ser. Prykladn. Mat. Inform., 7 (2003), 86-96. (in Ukrainian)

5. Senio P.S., Two-sided approximation finding of a function based on mathematics of functional intervals. Proceedings of XXXII International Conference PDMU. Czech Republic. Prague. August 27-31, 2018, 146-154.

6. Ilin V.A., Sadovnichiy V.A., Sendov B.K., Mathematical Analysis. Beginner course. M.: MGU, 1985. (in Russian)

7. Schwartz L., Analysis, V.1, M.: Mir, 1972. (in Russian)

8. Zorich V.A., Mathematical Analysis II. Berlin-Heidelberg: Springer-Verlag, 2 ed., 2016.

Ivan Franko National University of Lviv

Lviv, Ukraine

senio.petro@gmail.com 